INPLASY

PROTOCOL

To cite: Li et al. Efficacy and safety of adalimumab in the treatment of Juvenile Idiopathic Arthritis-Associated Uveitis: a meta-analysis and systematic review. Inplasy protocol 2020110002. doi: 10.37766/inplasy2020.11.0002

Received: 01 November 2020

Published: 01 November 2020

Corresponding author: Biao Li

2037276404@qq.com

Author Affiliation:

Teaching hospital of Chengdu University of Traditional

Chinese Medicine

Support: Province Department of science.

Review Stage at time of this submission: The review has not yet started.

Conflicts of interest: None.

\section{Efficacy and safety of adalimumab in the treatment of Juvenile Idiopathic Arthritis-Associated Uveitis: a meta- analysis and systematic review}

\author{
Li, B1; Li, H²; Zhang, L3; Zheng, Y4.
}

Review question / Objective: P: patients aged 2 to 18 years with Juvenile Idiopathic Arthritis-Associated Uveitis; I: adalimumab; C: Conventional routine treatment; $\mathrm{O}$ : Control of inflammation; Best-corrected visual acuity (BCVA); S: randomized controlled trials (RCTs).

Condition being studied: Uveitis is a potentially visionthreatening extra-articular manifestation of juvenile idiopathic arthritis (JIA) that manifests in approximately $13 \%$ of all patients. According to the national ophthalmological and paediatric rheumatological database, one out of four children with JIA and uveitis develops ocular complications such as synechiae, band keratopathy, cataract, glaucoma and macula oedema. The cumulative incidence of uveitis in JRA varies according to geographic location, presence of ANA, type of JRA onset and gender. Topical glucocorticoids should be used as initial treatment to achieve control of inflammation. Methotrexate and the monoclonal antibody tumor necrosis factor inhibitors adalimumab is recommended when systemic treatment is needed for the management of uveitis. The timely addition of adalimumab is recommended to maintain uveitis control in children who are at continued risk of vision loss.

INPLASY registration number: This protocol was registered with the International Platform of Registered Systematic Review and Meta-Analysis Protocols (INPLASY) on 01 November 2020 and was last updated on 01 November 2020 (registration number INPLASY2020110002).

\section{INTRODUCTION}

Review question / Objective: P: patients aged 2 to 18 years with Juvenile Idiopathic Arthritis-Associated Uveitis; I: adalimumab
C: Conventional routine treatment; 0 : Control of inflammation; Best-corrected visual acuity (BCVA); S: randomized controlled trials (RCTs). 
Rationale: To summarize updated evidences on the efficacy and safety of adalimumab in the treatment of patients with Juvenile Idiopathic ArthritisAssociated Uveitis.

Condition being studied: Uveitis is a potentially vision-threatening extraarticular manifestation of juvenile idiopathic arthritis (JIA) that manifests in approximately $13 \%$ of all patients. According to the national ophthalmological and paediatric rheumatological database, one out of four children with JIA and uveitis develops ocular complications such as synechiae, band keratopathy, cataract, glaucoma and macula oedema. The cumulative incidence of uveitis in JRA varies according to geographic location, presence of ANA, type of JRA onset and gender. Topical glucocorticoids should be used as initial treatment to achieve control of inflammation. Methotrexate and the monoclonal antibody tumor necrosis factor inhibitors adalimumab is recommended when systemic treatment is needed for the management of uveitis. The timely addition of adalimumab is recommended to maintain uveitis control in children who are at continued risk of vision loss.

\section{METHODS}

Search strategy: A relevant literature search will be conducted using the electronic databases of PubMed, Web of Seience, EMBASE, Cochrane, Library, clinicaltrials, CBM, CNKI, Wanfang and VIP from their inception to November 19, 2020, with no language restriction. We will use the following search terms: "juvenile idiopathic arthritis" or "Juvenile Rheumatoid Arthritis" or "Juvenile Arthritis" ; crossed with Interferonalpha,Interferon alpha, alpha-Interferon, Interferon, Lymphoblastoid Interferon, Lymphoblast Interferon. The search was restricted in the abstract/keywords/title fields to cut down on irrelevant literature. Study types were limited to RCTs.

Participant or population: Participants aged 2 to 18 years of gender or ethnicity with a diagnosis of Juvenile Idiopathic Arthritis-
Associated Uveitis; Participants received adalimumab for the treatment of Juvenile Idiopathic Arthritis-Associated Uveitis; The uveitis inflammatory activity grading was described according to the Standardization of UveitisNomenclature (SUN) workinggroup criteria; At least a 3-month median follow-up duration; Studies with at least five patients to avoid a positive report bias.

Intervention: Received adalimumab for the treatment of Juvenile Idiopathic ArthritisAssociated Uveitis.

Comparator: Conventional routine treatment (such as local and systemic corticosteroids, immunosuppressants).

Study designs to be included: Randomised controlled trials (RCTs).

Eligibility criteria: Study design § Randomised controlled trials (RCTs); Participants § Participants aged 2 to 18 years of gender or ethnicity with a diagnosis of Juvenile Idiopathic ArthritisAssociated Uveitis; Studies on a population broader than Juvenile Idiopathic ArthritisAssociated Uveitis will only be included if data specific for the Non-infectious uveitis subgroup is reported separately. The uveitis inflammatory activity grading was described according to the Standardization of Uveitis Nomenclature (SUN) workinggroup criteria; at least a 3-month median follow-up duration; studies with at least five patients to avoid a positive report bias. patients received adalimumab for the treatment of Juvenile Idiopathic ArthritisAssociated Uveitis.

Information sources: A relevant literature search will be conducted using the electronic databases of PubMed + Web of Science + EMBASE + Cochrane Library + clinicaltrials, CBM + CNKI + Wanfang, and VIP. From January 2000 to September 2017, with no language restriction. The terms/key words were "juvenile idiopathic arthritis" or "Juvenile Rheumatoid Arthritis" or "Juvenile Arthritis". Crossed with "anti-TNF a" or "anti-tumor necrosis factor alpha" or "anti TNF alpha" or "TNF alpha inhibitors" 
and "adalimumab". Study types were limited to RCTs.

Main outcome(s): Intraocular inflammation remission * Measures of effect $\S$ Weighted Mean Difference (WMD) intraocular inflammation is defined by the anterior chamber cells and/or vitreous haze decreasing by two levels or to grade $0.5+$ or 0 , respectively, according to the Standardization of Uveitis Nomenclature working group(SUN) criteria and National Eye Institute system criteria adopted by SUN in at least one eye. Besides, some studies require structural complications or comorbidities, such as cystoid macular edema (CME), vasculitis, and papillary edema, to be confirmed by fluorescein angiography (FA) or optical coherence tomography (OCT) examinations. For a comprehensive analysis, we included all of these studies.

Additional outcome(s): 1.Best-corrected visual acuity (BCVA); 2.corticosteroidsparing effect; 3.Central Macular Thickness (CMT); 4.Adverse events * Measures of effect $\S 1 \sim 3:$ Weighted Mean Difference (WMD) § 4: Adverse events: relative risks (RR).

Data management: Two authors will independently extract data from the included publications. Any discrepancies will be resolved through discussion and referral to a third reviewer if needed. A standardised piloted data extraction form will be used. Study authors may be contacted if further information is required. For each study, the following information (but not limited to) will be extracted. Study characteristics § Authors-publication year - title and journal § Study design § Setting § Sample size § Length of follow-up § Analysis. Participant characteristics § Patient selection/recruitment criteria § Patients' characteristics (demographic data, number, age, gender, socioeconomic status and ethnicity). § Type of uveitis (anatomical categorisation, syndrome/ aetiological classification) § Comorbidity § Co-medication Intervention and comparator $\S$ agents $\S$ Regimen (dose, frequency of administration, route of administration) Outcomes and findings $\S$ Outcomes measured and results for each outcome including precision and statistical test results.

Quality assessment / Risk of bias analysis: Quality assessment of all included articles will be under-taken by two reviewers independently with disagreements resolved by discussion and referral to a third reviewer if required. We will independently assess the risk of bias as described in the Cochrane Handbook for Systematic Reviews of Interventions. The following items will be assessed: random sequence generation; allocation concealment; blinding; incomplete outcome data; selective outcome reporting; other bias. In case of any unresolved disagreements, MSL will make the final decision as arbiter.

Strategy of data synthesis: All statistical analyses will be performed using Review Manager software (version 5.3). Continuous data will be reported as mean difference with $95 \%$ Cls. For primary outcomes, if the meta-analysis results are significantly heterogeneous, subgroup analysis will be performed as detailed below. We will use the Grading of Recommendations Assessment, Development and Evaluation (GRADE) software to determine the quality of evidence based on the Cochrane Handbook for Systematic Reviews of Interventions to create a Summary of Findings table. We will use the random effect model for meta-analysis. If a metaanalysis is possible, we will use the $I^{2}$ statistic to quantify inconsistencies across the included studies. A resulting $50 \%$ cut-off point would represent substantial heterogeneity. Where heterogeneity is exited, we will conduct subgroup analyses.

Subgroup analysis: Where studies and data permit, subgroup analyses will be conducted according to the types of uveitis, duration of treatment or patients' ages.

Sensibility analysis: In the case of sufficient trials data, the risk of bias tool will be used to assess methodological quality. If low- 
quality articles are deleted, a second metaanalysis will be performed. The results and effect size of the two meta-analyses will be compared and discussed.

Language: No language restriction.

Country(ies) involved: China.

Keywords: Juvenile Idiopathic ArthritisAssociated Uveitis; adalimumab; efficacy; safety; randomized controlled trials.

Contributions of each author:

Author 1 - Biao Li - Conceptualization; Data curation; Formal analysis; Methodology; Writing - original draft; Writing - review \& editing.

Author 2 - Haoran Li - Data curation; Methodology; Writing- original draft.

Author 3 - Li Zhang - Formal analysis; Methodology; Writing - original draft.

Author 4 - Yanlin Zheng - Project administration; Supervision; Writing review \& editing. 